\title{
A Prognostic Model of Pontine Hemorrhage Based on Hemorrhage Volume and Location
}

\author{
Minhan Kim ${ }^{1}$, Suhee $\mathrm{Cho}^{3}$, Seung Hoon You ${ }^{3}$, Jieun Park ${ }^{4}$, Wonhyoung Park ${ }^{1}$, Jung Cheo Park ${ }^{1}$, Jae Sung \\ Ahn $^{1}$, Moinay Kim ${ }^{1,2}$, Seungjoo Lee ${ }^{1,2}$ \\ ${ }^{1}$ Department of Neurological Surgery, Seoul Asan Medical Center, University of Ulsan College of Medicine, Seoul, Korea \\ ${ }^{2}$ Divsion of Neurosurgical Critical Care, Department of Neurosurgery, Asan Medical Center, University of Ulsan College of Medicine, Seoul, Korea \\ ${ }^{3}$ Department of Neurological Surgery, Gangneung Asan Medical Center, University of Ulsan College of Medicine, Gangneung, Korea \\ ${ }^{4}$ Department of Radiology, Asan Medical Center, University of Ulsan College of Medicine, Seoul, Korea
}

Received: March 11, 2021

Accepted: April 1, 2021

\section{Corresponding Author:}

Seungjoo Lee, M.D., Ph.D., Department of Neurological Surgery, Asan Medical Center, University of Ulsan College of Medicine, 43gil Olympic-ro, Songpa-gu, Seoul 05505, Korea.

Fax: 82-2-476-6738

Tel: 82-2-3010-3550

E-mail: aussie84@naver.com; changhill@gmail.com

Co-Corresponding Author: Moinay Kim, M.D., Ph.D., Department of neurological surgery, Asan Medical Center, University of Ulsan College of Medicine, 43gil Olympic0ro, Songpa-gu, Seoul 05505, Korea Fax: 82-2-476-6738

Tel: 82-2-3010-1381

Email: aussie84@naver.com

\section{Objective}

Spontaneous pontine hemorrhages have the highest mortality rate. Posterior pontine hemorrhage has a poor prognosis, although more research is required in this area. Herein, we attempted to determine the prognosis of pontine hemorrhage by schematically illustrating the volume and location and developing a scoring model to predict the relationship between initial hemorrhage characteristics and clinical outcome.

\section{Methods}

This multicenter, retrospective study was conducted between January 2010 and December 2019. We developed a scoring model using computed tomography (5-mm sections) to plot the location and volume of pontine hemorrhages. All hemorrhage volumes were classified as mild $\left(0.5-5 \mathrm{~cm}^{3}\right)$, moderate $\left(5-10 \mathrm{~cm}^{3}\right)$, or severe $\left(10-16 \mathrm{~cm}^{3}\right)$.

Results

As the pontine hemorrhage volume increased, the Glasgow Coma Scale (GCS) and Eastern Cooperative Oncology Group scores (ECOG) were significantly lower, while the modified Rankin Scale (mRS) was higher $(\mathrm{p}<0.001)$. In the mild and moderate groups, the GCS recovered from 11.36 to 12.89 and 4.68 to 7.31 over 24 months, respectively. The mRS improved from 3.25 to 2.82 in the mild hemorrhage group but deteriorated in the moderate hemorrhage group from 4.93 to 5.24 over 24 months. More extensive pontine hemorrhages were associated with shorter life expectancy. The mild, moderate, and severe groups showed $32.79 \%$, $89.29 \%$, and $100 \%$ mortality, respectively $(\mathrm{p}<0.0001)$. The anterior and posterior hemorrhage groups had $69.44 \%$ and $42.86 \%$ mortality $(\mathrm{p}=0.0020)$, respectively.

\section{Conclusion}

According to our prognosis model, initial hemorrhage volume was the most significantly related neurological outcome. Hemorrhage location showed no relationship with neurological outcome. However, anterior upper pontine hemorrhage volume was correlated with shortened survival time.

Keywords: Prognostic model of pontine hemorrhage; Prognosis of pontine hemorrhage; Anterior and posterior pontine hemorrahge; Neurologic outcome of pontine hemorrhage 


\section{INTRODUCTION}

The brainstem consist of the midbrain, the pons, and the medullar oblongata, located in the posterior part of the brain ${ }^{1)}$. The brainstem is a unique structure and has the crucial connection among the cerebrum, the cerebellum and the spinal cord. The brainstem is responsible for multiple critical functions, including blood pressure, heart rate, respiration and awakeness ${ }^{2}$. The cranial nerve nuclei that are located in the brainstem have a crucial role in balance, hearing, swallowing, speech, motor and sensory. In other words, brainstem injury can result in various symptoms like cardiac and respiratory dysfunction, facial palsy, dysarthria, vision abnormality, decreased consciousness and even death. Hence early diagnosis and appropriate management of brainstem injury is the key factor to reduce morbidity and mortality.

Hemorrhagic stroke is a disastrous cerebrovascular disease associated with a vast degree of morbidity and mortality ${ }^{3)}$. Among these, intracerebral hemorrhage (ICH) accounts for $10-28 \%$ of stroke worldwide and results in very high mortality and morbidity rates that have not changed for more than decades ${ }^{4)}$.

Pontine hemorrhage accounts for approximately $10 \%$ of all ICH5), and its outcome is closely related to the recovery of consciousness, motor function, and respiratory drive as the cranial nerves V to VIII originate from the pons ${ }^{6}$. . These patients usually have very poor prognosis with overall mortality rates of $30-50 \%{ }^{3)}$. Despite these high mortality rates, most of pontine hemorrhage patients are treated conservatively.6 For primary hemorrhage originate from the posterior fossa, the American Stroke Association Guidelines recommend considering the surgical removal of cerebellar hematoma, but not the brainstem hematoma ${ }^{7)}$. The guideline recommends against the surgical evacuation of brainstem hematoma for poor clinical outcome. Hence, it is difficult to treat and predict the outcome of brainstem hemorrhage patients.

Several studies have attempted to predict prognosis in patients with pontine hemorrhage, although many predictors based on clinical and radiological features have been identified in conservative patients ${ }^{6,8)}$. Previous studies have found that brainstem volume is similar in adult patients, regardless of age ${ }^{9)}$, and the greater hemorrhage volume is associated with reduced consciousness and worse prognosis, regardless of age or $\operatorname{sex}^{6,8,10,11)}$. Few reports have revealed an association between prognosis and bleeding location $^{5,12-18)}$. These reports show large variation in survival and functional recovery rates.

In the present study, we exclusively developed a pontine hemorrhage measurement model using an axial cut in a simple, 5-mm computed tomography (CT) scan. We hypothesized that prognosis in patients with pontine hemorrhage could be predicted by schematically illustrating the hemorrhage's volume and location. On this basis, we developed a scoring model to predict the relationship between initial pontine hemorrhage characteristics and clinical outcome. To the best of our knowledge, no report has focused on the association between the volume and location of pontine hemorrhage with patient's clinical outcome.

\section{MATERIALS AND METHODS}

Appropriate institutional review board approval and patient consent were obtained for this study. The data that support the findings of this study are available from the corresponding author upon reasonable request.

\section{Study Design and Participants}

This is a retrospective and multicenter study conducted in different Korean neuro-intensive care units (ICU)s between January 2010 and December 2019. Only patients aged over 18 years were included in the study. The following are criteria for inclusion of patients in our study: 1) diagnosis of pontine ICH based on CT including hypertensive, vascular abnormality (arteriovenous malformation $[\mathrm{AVM}]$, cavernous malformation $[\mathrm{CM}]$, or malignancy bleeding, 2) age $\geq 18$ or $\leq 80$ years; and 3 ) expectation of staying in the ICU $\geq 48$ hours. The exclusion criteria were as follows: (1) any evidence of traumatic brain injury, (2) loss to follow-up before 24-months post-hemorrhage (3) pregnancy, (4) post-cardiac arrest with the diagnosis of severe hypoxic brain injury, and (5) do not resuscitate ordered patients. The relevant data were obtained from the medical records by a physician and trained research nurse. The following candidate factors were analyzed: (1) age, (2) sex, (3) comorbidities, namely hypertension (previous diagnosis or prescription of relevant medication), diabetes mellitus (previous diagnosis or prescription of relevant medication), end-stage renal disease (prior diagnosis of chronic kidney disease or regular hemodialysis), the vascular abnormality (AVM, CM or etc.), malignancy bleeding, previous cancer history, (4) hemorrhage date, and (5) use of anticoagulants or antiplatelets (Table 1).

\section{Radiologic Evaluation}

The diagnosis of ICH was assessed based on CT scans. The volume of the brainstem in males and females does not differ significantly among various age groups ${ }^{9)}$. Accordingly, we developed a scoring model using a CT scan (5-mm thin section) (Fig. 1). In this model, we defined a single cell as a hemorrhage volume of 0.5 $\mathrm{cm}^{3}(8 \times 7.5 \times 5 \mathrm{~mm})$. All hemorrhages were classified as mild (0.5-5 cm ; $1-10$ colored cells), moderate $\left(5-10 \mathrm{~cm}^{3} ; 11-20\right.$ colored cells), or severe $\left(10-16 \mathrm{~cm}^{3} ; 21-32\right.$ colored cells). All pa- 
Table 1. Table caption

\begin{tabular}{|c|c|c|c|c|}
\hline Characteristics & Mild (n=61) & Moderate $(n=28)$ & Severe $(n=12)$ & p-value \\
\hline Sex (Male:Female) (\%) & $3.7: 1$ & $2.2: 1$ & $5: 1$ & 0.0435 \\
\hline Age \pm & $56.4( \pm 12.4)$ & $59.9( \pm 11.7)$ & $59.4( \pm 17.6)$ & 0.4441 \\
\hline \multicolumn{5}{|l|}{ Admission } \\
\hline $\mathrm{sBP} \pm$ & $170.5( \pm 42.0)$ & $170.7( \pm 31.2)$ & $176.3( \pm 35.0)$ & 0.9128 \\
\hline $\mathrm{dBP} \pm$ & $100.0( \pm 27.5)$ & $97.9( \pm 27.4)$ & $101.0( \pm 27.9)$ & 0.9376 \\
\hline $\mathrm{mBP} \pm$ & $123.5( \pm 30.1)$ & $122.2( \pm 25.3)$ & $126.1( \pm 29.4)$ & 0.9380 \\
\hline $\mathrm{BST} \pm$ & $146.9( \pm 52.2)$ & $183.9( \pm 68.8)$ & $209.6( \pm 74.5)$ & 0.0014 \\
\hline \multicolumn{5}{|l|}{ Underlying ds. (\%) } \\
\hline HTN & $43(70.5 \%)$ & $14(50.0 \%)$ & $8(66.7 \%)$ & 0.1685 \\
\hline $\mathrm{DM}$ & $8(13.1 \%)$ & $4(14.3 \%)$ & $4(33.3 \%)$ & 0.2110 \\
\hline Vascular lesion & $4(6.6 \%)$ & $3(10.7 \%)$ & $0(0 \%)$ & 0.6154 \\
\hline ESRD & $4(6.5 \%)$ & $4(14.3 \%)$ & $1(8.3 \%)$ & 0.4601 \\
\hline $\mathrm{CM}$ & $9(14.8 \%)$ & $2(7.1 \%)$ & $0(0 \%)$ & 0.3666 \\
\hline Metastatic brain tumor & $2(3.3 \%)$ & $0(0 \%)$ & $0(0 \%)$ & 1.0000 \\
\hline Perviouscancer history & $10(16.4 \%)$ & $4(14.3 \%)$ & $0(0 \%)$ & 0.3753 \\
\hline Antiplatelets and anticoagulants use & $6(9.8 \%)$ & $5(17.9 \%)$ & $3(25 \%)$ & 0.2244 \\
\hline
\end{tabular}

sBP: Systolic blood pressure, dBP: Diastolic blood pressure, mBP: Mean blood pressure, ds.: disease, HTN: Hypertension, DM: Diabetes mellitus, ESRD: End-stage renal disease, CM: Cavernous malformation, Hemorrhage volume: Mild, $1-10$ colored cells $\left(0.5-5 \mathrm{~cm}^{3}\right)$, moderate: $11-20$ colored cells $(5-10$ $\left.\mathrm{cm}^{3}\right)$, severe: $21-32$ colored cells $\left(10-15 \mathrm{~cm}^{3}\right)$.

*Aspirin, plavix, cilostazol, warfarin, novel oral anticoagulants; \pm : these values are given am mean \pm standard deviation.

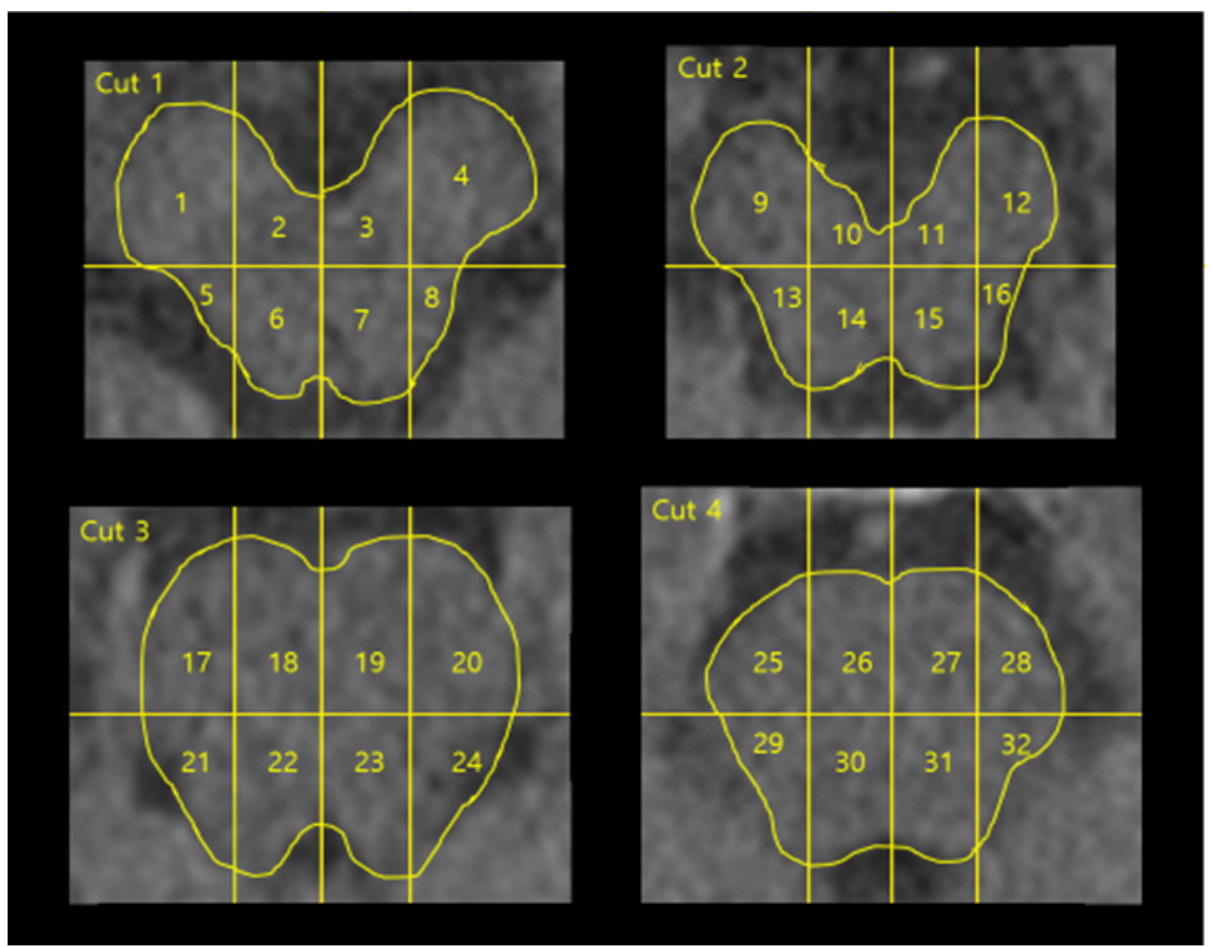

Fig. 1. Schematic tool for pontine hemorrhage volume estimation.

tients' schematic data, and each cell was numbered and converted into data. In the location analysis, cells numbered 1-4, 9-12, 1720 , or 25-28 were classified as anterior, while all others were defined as posterior. Cells numbered 1-16 were classified as mid- brain, while all others were defined as pontine. The volume of hemorrhage was measured with CT using 1.5 to $5 \mathrm{~mm}$-thick slices; the slices were processed using software developed in-house [AsanJ, based on a plug-in package ImageJ (https://rsbweb.nih. 
gov/ij)]. To minimize measurement errors, the hemorrhage measurements were performed by two board-certified neurosurgeons who were blinded to treatment assignment and the mean value was documented.

\section{Assessment and Follow-up}

All patients were assessed using the Glasgow Coma Scale (GCS), Eastern Cooperative Oncology Group performance status (ECOG), and modified Rankin Scale (mRS) at 0, 6, 12, and 24 months from the bleeding date. If not possible, patient evaluation was performed via the telephone interview by a nurse or doctor not involved in the study and patient's management. The primary outcome was to evaluate the clinical relationship between the hemorrhage volume, location with our assessment tools on different time intervals.

\section{Statistical Analysis}

All data were summarized as mean \pm standard deviation for continuous variables and frequency and percentage for categorical variables. Hemorrhage volume groups were compared using either the chi-squared or Fisher's exact test for categorical data, and either analysis of variance or the Kruskal-Wallis test for continuous variables. To analyze longitudinally measured outcomes, such as GCS, ECOG, and mRS, a linear mixed model was applied to the evaluated group; least square mean, and standard error were used to evaluate time effect, as well as how group and time interacted with outcomes. Crude Cox's proportional hazard model was used to evaluate how hemorrhage volume and location were associated with death, while Cox's proportional hazard model was applied for multivariable adjustments. Statistical analysis was carried out using SAS 9.4 (SAS Institute, Cary, NC). A p-value < 0.05 was deemed statistically significant.

\section{RESULTS}

We retrospectively screened 190 hemorrhagic stroke patients who were admitted to ICU between January 2010 and December 2019. After inclusion and exclusion criteria were applied, we retrospectively identified 101 patients. There were no significant differences in baseline characteristics between the patients with different hemorrhage volumes except the blood sugar level at admission (Table 1). The mean age for the mild, moderate and severe groups were $56.4 \pm 12.4,59.9 \pm 11.7,59.4 \pm 17.6$ years, respectively. All groups showed male predominance $(\mathrm{p}=0.0435)$. The most common comorbidities were hypertension (Mild $70.5 \%$ vs. Moderate $50 \%$ vs. Severe $66.7 \%, \mathrm{p}=0.1685$ ) and diabetes (Mild $13.1 \% \%$ vs. Moderate $14.3 \%$ vs. Severe $33.3 \%, p=0.2110$ ). Previous adminis- tration of antiplatelet or anticoagulant showed no significant differences between the three groups (Mild 9.8\% vs. Moderate $17.9 \%$ vs. Severe $25 \%, \mathrm{p}=0.2244$ ). The results are summarized in Table 1 .

The significant relationship between the neurological outcomes and hemorrhage volume was observed (Table 2; Fig. 2). On admission GCS was significantly different among 3 groups (Mild $11.36 \pm 0.47$ vs. Moderate $4.68 \pm 0.69$ vs. Severe $3.08 \pm 1.05$, $\mathrm{p}<0.0001)$. GCS evaluated 3-month after the hemorrhage showed similar results; Mild $12.54 \pm 0.41$ vs. Moderate $6.16 \pm 0.72$, $\mathrm{p}<0.0001$. No significant differences were observed on 12 and 24-month GCS evaluation. Severe group patients were not included in the 6,12 and 24-month evaluation as no patients survived at this time interval. The significant difference was observed comparing the admission and 24-month GCS improvement (Mild $1.53 \pm 0.58$ vs. Moderate $2.64 \pm 1.94, p=0.0001$ ). Similar findings were observed for the clinical outcomes using ECOG and $\mathrm{mRS}$.

Unlike the strong association between the hemorrhage volume and the neurological outcome, significant association was only observed on only time effect assessment in the hemorrhage location analysis (Table 3 ). When the location was dichotomized into anterior and posterior, mild $(\mathrm{p}=0.0277)$ and moderate $(\mathrm{p}=0.0002)$ groups showed significant differences at time effect. No other significant findings were observed on group effect, interaction of group \& time, group comparisons at a specific time point 0 and 0 vs 24 months. Similar findings were observed when the hemorrhage locations were dichotomized into upper and lower pontine locations. The significant association was only observed on only time effect in the $\mathrm{mRS}$ for mild $(\mathrm{p}=0.0459)$ and moderate $(\mathrm{p}=0.0025)$ groups. No other significant findings were observed on any other effect and group comparisons. Severe group patients $(n=5)$ were not included in the location analysis as there were too small number of patients to perform statistical analysis.

In terms of life expectancy, a significant difference was found depending on the location and volume of pontine hemorrhage (Table 4; Fig. 2). Larger pontine hemorrhage was related to shorter life expectancy. The 24-month follow-up mortality rates in the mild, moderate, and severe groups were $32.79 \%, 89.29 \%$, and $100 \%$, respectively $(\mathrm{p}<0.0001)$. In the location analysis, there was a positive correlation between anterior pontine bleeding volume and shortened survival time. In the multivariable-adjusted analysis, the anterior and posterior hemorrhage group had $69.44 \%$ and $42.86 \%$ mortality rates, respectively, after adjustment for hemorrhage volume ( $\mathrm{p}=0.0020)$ (Fig. 2). In the midbrain and pontine groups, the mortality rates were $57.14 \%$ and $52.94 \%$, respectively. The hazard ratio $(\mathrm{HR})$ was high $(\mathrm{HR}=1.518)$, but no significant results were obtained $(\mathrm{p}=0.5867)$ because the midbrain hemorrhage group had only four patients. 
Table 2. Relationship between neurological outcomes and hemorrhage volume over time

\begin{tabular}{|c|c|c|c|c|c|}
\hline & Months & Mild (n=61) & Moderate $(\mathrm{n}=28)$ & Severe $(n=12)$ & p-value \\
\hline \multirow[t]{5}{*}{ GCS score \pm} & 0 & $11.36( \pm 0.47)$ & $4.68( \pm 0.69)$ & $3.08( \pm 1.05)$ & $<0.0001^{*}$ \\
\hline & 6 & $12.54( \pm 0.41)$ & $6.16( \pm 0.72)$ & & $<0.0001^{* *}$ \\
\hline & 12 & $12.95( \pm 0.39)$ & $7.02( \pm 0.74)$ & & $<0.0001^{* *}$ \\
\hline & 24 & $12.89( \pm 0.55)$ & $7.31( \pm 1.93)$ & & $0.4547^{* *}$ \\
\hline & 0 vs. 24 & $+1.53( \pm 0.58)$ & $+2.64( \pm 1.94)$ & & $0.0001^{*}$ \\
\hline \multirow[t]{5}{*}{$\mathrm{ECOG} \pm$} & 0 & $2.54( \pm 0.13)$ & $3.89( \pm 0.19)$ & $4.00( \pm 0.29)$ & $<0.0001^{*}$ \\
\hline & 6 & $2.15( \pm 0.14)$ & $3.63( \pm 0.26)$ & & $<0.0001^{* *}$ \\
\hline & 12 & $2.04( \pm 0.14)$ & $3.63( \pm 0.28)$ & & $0.3482^{* *}$ \\
\hline & 24 & $2.05( \pm 0.14)$ & $3.15( \pm 0.30)$ & & $<0.0001^{* *}$ \\
\hline & 0 vs. 24 & $-0.49( \pm 0.10)$ & $+0.74( \pm 0.26)$ & & $0.6583^{*}$ \\
\hline \multirow[t]{5}{*}{$\mathrm{mRS} \pm$} & 0 & $3.25( \pm 0.21)$ & $4.93( \pm 0.31)$ & $5.08( \pm 0.48)$ & $<0.0001^{*}$ \\
\hline & 6 & $3.15( \pm 0.21)$ & $5.59( \pm 0.32)$ & $6.00( \pm 0.48)$ & $<0.0001^{* *}$ \\
\hline & 12 & $2.93( \pm 0.22)$ & $5.82( \pm 0.41)$ & & $<0.0001^{* *}$ \\
\hline & 24 & $2.82( \pm 0.23)$ & $5.24( \pm 0.56)$ & & $<0.0001^{* *}$ \\
\hline & 0 vs. 24 & $-0.43( \pm 0.13)$ & $0.32( \pm 0.49)$ & & $<0.0001^{*}$ \\
\hline
\end{tabular}

GCS: Glasgow coma scale, ECOG: Eastern cooperative oncology group performance status, mRS: modified Rankin Scale.

p value of group comparisons at a specific time point 0,0 vs. 24 months respectively.

linear mixed model: $\mathrm{p}$-value of group effect, time effect and interaction of group \& time respectively \pm : these values are given as mean \pm standard deviation.
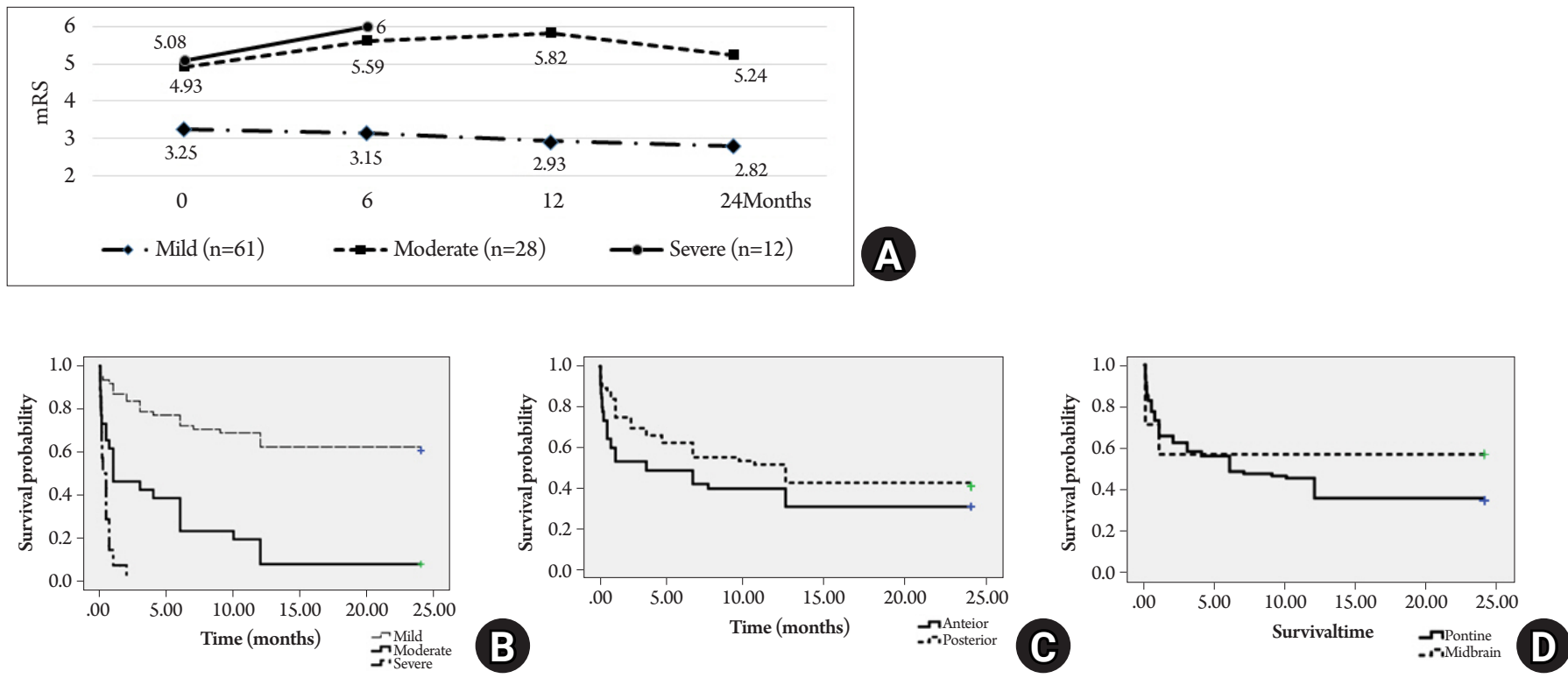

Fig. 2. (A) The relationship between mRS and hemorrhage volume over time. (B) Survival time in the each amount of hemorrhage groups (mild, moderate and severe), (C,D) anterior vs posterior and midbrain vs pontine hemorrhage groups.

\section{DISCUSSION}

Spontaneous pontine hemorrhage is becoming more common and has a significant impact on the physical, psychological, and social well-being of patients and their families. Some patients recover neurologically, while others have a poor neurological progno$\operatorname{sis}^{5,11,12,19)}$. In general, posterior pontine hemorrhage has a poorer prognosis than anterior ${ }^{18)}$, but few studies have investigated this difference, and the present study showed contradictory results in this regard.

We decided to use a simple schematic tool to quantify pontine hemorrhage. Human brainstem volume is almost constant in males and females, at about $18.99 \pm 2.36 \mathrm{~cm}^{3}$ and $22.05 \pm 4.01 \mathrm{~cm}^{3}$, respectively. Moreover, it does not differ significantly with age ${ }^{9)}$. Therefore, the number of 5-mm brainstem cuts seen in an axial CT scan is probably constant. We schematically illustrated pontine 
Table 3. Anterior vs posterior and midbrain vs pontine location analysis with $\mathrm{mRS}$ score $(0,6,12,24$ months $)$

\begin{tabular}{|c|c|c|c|c|c|c|c|}
\hline \multirow{2}{*}{ Hemorrhage volume } & \multirow{2}{*}{ Months } & \multicolumn{2}{|c|}{$\mathrm{mRS} \pm$} & \multirow{2}{*}{ p-value } & \multicolumn{2}{|c|}{$\mathrm{mRS} \pm$} & \multirow{2}{*}{ p-value } \\
\hline & & Anterior & Posterior & & Midbrain & Pontine & \\
\hline Mild & 0 & $3.43( \pm 0.44)$ & $3.21( \pm 0.33)$ & 0.6512 & $1.67( \pm 1.00)$ & $3.35( \pm 0.23)$ & 0.1068 \\
\hline$(\mathrm{n}=60)^{*}$ & 6 & $3.38( \pm 0.44)$ & $3.08( \pm 0.33)$ & $0.6246^{*}$ & $0.67( \pm 1.25)$ & $3.27( \pm 0.29)$ & $0.0548^{*}$ \\
\hline \multirow[t]{3}{*}{$(\mathrm{n}=59)^{*}$} & 12 & $3.09( \pm 0.46)$ & $2.91( \pm 0.34)$ & $0.0277^{*}$ & $0.67( \pm 1.47)$ & $3.57( \pm 0.35)$ & $0.0459^{*}$ \\
\hline & 24 & $3.09( \pm 0.46)$ & $2.73( \pm 0.35)$ & $0.9288^{*}$ & $0.67( \pm 1.55)$ & $3.39( \pm 0.46)$ & $0.4986^{*}$ \\
\hline & 0 vs. 24 & $-0.34( \pm 0.24)$ & $-0.48( \pm 0.18)$ & 0.4892 & $-1.00( \pm 1.04)$ & $+0.34( \pm 0.25)$ & 0.7481 \\
\hline \multirow[t]{4}{*}{$(\mathrm{n}=28)^{*}$} & 6 & $5.70( \pm 0.26)$ & $5.47( \pm 0.23)$ & $0.1936^{*}$ & $6.00( \pm 0.62)$ & $5.55( \pm 0.17)$ & $0.6669^{*}$ \\
\hline & 12 & $5.75( \pm 0.26)$ & $5.32( \pm 0.44)$ & $0.0002^{*}$ & & $5.77( \pm 0.26)$ & $0.0025^{*}$ \\
\hline & 24 & $5.55( \pm 0.58)$ & $4.76( \pm 0.58)$ & $0.7250^{*}$ & & $5.17( \pm 0.40)$ & $0.4392^{*}$ \\
\hline & 0 vs. 24 & $+0.46( \pm 0.55)$ & $-0.04( \pm 0.56)$ & 0.7934 & & $+0.24( \pm 0.38)$ & 0.7934 \\
\hline Severe & 0 & & & & & & \\
\hline$(n=5)^{+}$ & 0 vs. 24 & & & & & & \\
\hline
\end{tabular}

The other was p value of group comparisons at a specific time point 0,0 vs. 24 months respectively.

"Anterior vs posterior: Patients with the same anterior and posterior hemorrhage volumes were excluded.

"Midbrain vs pontine: Patients with the same anterior and posterior hemorrhage volumes were excluded.

"*** Linear mixed model, p-value of group effect, time effect, interaction of group \& time respectively.

${ }^{+}$The severe hemorrhage group had no statistics results because only five patients were included.

\pm : these values are given as mean \pm standard deviation; mRS: modified Rankin Scale.

Table 4. Mortality rate at 24 months

\begin{tabular}{lccc}
\hline Hemorrhage volume & Death $\mathrm{n}(\%)$ in 24 months & HR $(95 \% \text { CI })^{*}$ & p-value \\
\hline Mild $(\mathrm{n}=61)$ & $20(32.8 \%)$ & 1.00 & $5.57(2.48-12.51)$ \\
Moderate $(\mathrm{n}=28)$ & $25(89.3 \%)$ & $11.68(4.12-33.16)$ & \\
Severe $(\mathrm{n}=12)$ & $12(100 \%)$ & 1.00 & $<0.0001$ \\
Location analysis 1 & & $0.35(0.18-0.68)$ & \\
$\quad$ Anterior & $25(69.4 \%)$ & 1.00 & 0.0020 \\
$\quad$ Posterior & $24(42.9 \%)$ & $1.52(0.34-0.85)$ \\
Location analysis & & & 0.5867 \\
$\quad$ Midbrain & $4(57.1 \%)$ & & \\
$\quad$ Pontine & $45(52.9 \%)$ & &
\end{tabular}

HR: Hazard ratio.

Cells numbered 1-4, 9-12, 17-20, and 25-28 were classified into the anterior portion, while all other cells were placed into the posterior portion. Cells numbered 1-16 were classified into the midbrain portion, while all others were placed into the pontine portion (Fig. 1).

"Multivariable adjusted analysis used. Adjustment factor was used, as in Table 1.

hemorrhage using axial CT images, in which the midbrain was completely separated from the thalamus and surrounded by the ambient cistern.

Several papers have described the relationship between location and volume of brainstem hemorrhage ${ }^{11,12,18,20-23)}$, but most drew no schematic conclusion about the location of pontine bleeding. Many investigations have predicted that only initial consciousness, measured using the GCS, has any significant effect on prognosis. The prognosis of dorsal pontine hemorrhage is poor in horizontal brainstem structures such as the tectum. However, the schematic tool used in the present article is very intuitive and could be used to distinguish between the upper and lower pons, as well as to identify the hemorrhage location using only a CT scan easily.

Our study had some limitations. Referral bias cannot be excluded as all participating centers were large, university-affiliated, tertiary care institutions. Consequently, our prediction tool may not represent the general population.

Furthermore, the mRS does not accurately reflect neurological improvement or deterioration. As the mRS increases from 1 to 5 , the patient's neurological condition worsens. However, a patient 


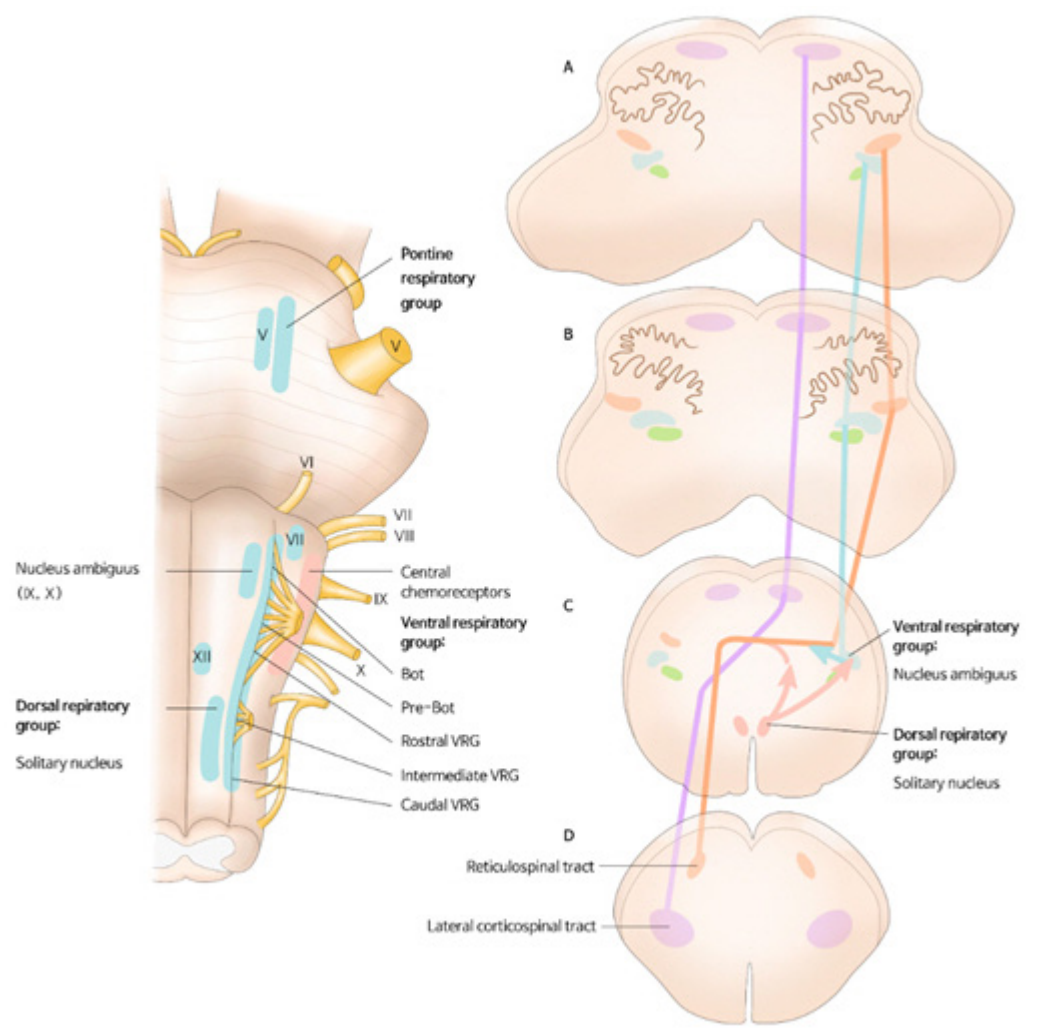

Fig. 3. Illustration of brain stem and major nuclei.

with 6 points on the mRS is considered dead, and it is impossible to determine whether their mortality was neurological in origin. Many pontine hemorrhages result in decreased consciousness and high mortality in the acute period because obstructive hydrocephalus leads to chemoreceptor injury and direct brainstem compres$\operatorname{sion}^{8,18,23)}$. Another limitation of the present study was that bleeding was only measured in the upper pons. There are three crucial brainstem respiratory centers: the pneumotaxic center or pontine respiratory group in the dorsal lateral pons, the dorsal respiratory group, and the ventral respiratory group (Fig. $3{ }^{24-26)}$. In the brainstem as a whole, the respiratory center is mainly in the rear, but it is concentrated in the front in the upper pons. In the present study, only upper pontine hemorrhage was analyzed, that is, from the midbrain to the point $2 \mathrm{~cm}$ lower. Therefore, the respiratory center would have been damaged in the group with a large volume of anterior pontine bleeding, and the respiratory tract was likely damaged in this group, influencing mortality. The correlation between location and prognosis in brainstem hemorrhage could be further studied in an analysis that included the pons and medulla.

Previous studies analyzing the prognosis of brainstem hemorrhage have been limited because they only analyzed the midbrain, pons, and medulla. However, in the present study, a more accurate bleeding-location relationship was confirmed using the intuitive schematic tool, which could subdivide the brain region. From this point of view, our study complemented previous papers' shortcomings and helped extend the data range.

\section{CONCLUSION}

According to our prediction model, the initial volume of pontine hemorrhage was most significantly related to neurological outcomes in the mild hemorrhage group. Hemorrhage location showed no relationship with neurological outcome. However, a positive correlation was observed between anterior upper pontine hemorrhage volume and shortened survival time. If the range of research data is extended from the lower pons to the medulla, the brainstem position with the most fatal prognosis could be evaluated using a simple CT scan.

\section{NOTES}

\section{Conflict of interest}

No potential conflict of interest relevant to this article was reported. 


\section{Funding}

This research was supported by the 2019IL0828, 2019IP0779 from the Asan Institute for Life sciences, Asan Medical Center (Seoul, Republic of Korea).

\section{Acknowledgements}

We thank Junghui Lee for providing the clinical data and Soyoung Jung for summarizing the clinical cases. We are also grateful to Prof. Jae Sung Ahn for his generous support in equipment and clinical resources. We thank the Magnetic Resonance Center facilities, the Computed Tomography Center at Asan Medical Center for their equipment, services, and expertise. Selective Plane Illumination Microscopy Light-sheet Z.1 was supported by the Brain Research Core Facilities at KBRI.

\section{REFERENCES}

1. Gowda SN, De Jesus O. Brainstem infarction. Statpearls. Treasure Island (FL): StatPearls Publishing Copyright (C) 2021, StatPearls Publishing LLC.; 2021.

2. Ortiz de Mendivil A, Alcalá-Galiano A, Ochoa M, Salvador E, Millán JM. Brainstem stroke: Anatomy, clinical and radiological findings. Semin Ultrasound CT MR 2013;34:131-141.

3. van Asch CJ, Luitse MJ, Rinkel GJ, van der Tweel I, Algra A, Klijn CJ. Incidence, case fatality, and functional outcome of intracerebral haemorrhage over time, according to age, sex, and ethnic origin: a systematic review and meta-analysis. Lancet Neurol 2010;9:167-176.

4. Kim M, Byun J, Chung Y, Lee SU, Park JE, Park W, et al. Reactive oxygen species scavenger in acute intracerebral hemorrhage patients: a multicenter, randomized controlled trial. Stroke 2021; Strokeaha120032266.

5. Behrouz R. Prognostic factors in pontine haemorrhage: a systematic review. Eur Stroke J 2018;3:101-109.

6. Sripontan S. Good outcome in a patient with massive pontine hemorrhage. Asian J Neurosurg 2019;14:992-995.

7. Hemphill JC, Greenberg SM, Anderson CS, Becker K, Bendok $\mathrm{BR}$, Cushman M, et al. Guidelines for the management of spontaneous intracerebral hemorrhage: a guideline for healthcare professionals from the american heart association/ american stroke association. Stroke 2015;46:2032-2060.

8. Tao C, Li H, Wang J, You C. Predictors of surgical results in patients with primary pontine hemorrhage. Turk Neurosurg 2016;26:77-83.

9. Erbagci H, Keser M, Kervancioglu S, Kizillkan N. Estimation of the brain stem volume by stereological method on magnetic resonance imaging. Surg Radiol Anat 2012;34:819-824.

10. Meguro T, Kuwahara K, Tomita Y, Okuma Y, Tanabe T, Muraoka K, et al. Primary pontine hemorrhage in the acute stage: Clinical features and a proposed new simple scoring system. Stroke Cerebrovasc Dis 2015;24:860-865.

11. Rabinstein AA, Tisch SH, McClelland RL, Wijdicks EF. Cause is the main predictor of outcome in patients with pontine hemorrhage. Cerebrovasc Dis 2004; 17:66-71.

12. Balci K, Asil T, Kerimoglu M, Celik Y, Utku U. Clinical and neuroradiological predictors of mortality in patients with primary pontine hemorrhage. Clin Neurol Neurosurg 2005; 108:36-39.

13. Huang K, Ji Z, Sun L, Gao X, Lin S, Liu T, et al. Development and validation of a grading scale for primary pontine hemorrhage. Stroke 2017;48:63-69.

14. Huang KB, Ji Z, Wu YM, Wang SN, Lin ZZ, Pan SY. The prediction of 30-day mortality in patients with primary pontine hemorrhage: A scoring system comparison. Eur J Neurol 2012;19:1245-1250.

15. Iwasaki Y, Kinoshita M, Ikeda K. Primary pontine hemorrhage: Clinico-computed tomographic correlations. Comput Med Imaging Graph 1988;12:365-370.

16. Jang JH, Song YG, Kim YZ. Predictors of 30-day mortality and 90-day functional recovery after primary pontine hemorrhage. J Korean Med Sci 2011;26:100-107.

17. Kim KH, Kim HD, Kim YZ. Comparisons of 30-day mortalities and 90-day functional recoveries after first and recurrent primary intracerebral hemorrhage attacks: A multiple-institute retrospective study. World Neurosurg 2013;79:489-498.

18. Wessels T, Möller-Hartmann W, Noth J, Klötzsch C. Ct findings and clinical features as markers for patient outcome in primary pontine hemorrhage. AJNR Am J Neuroradiol 2004;25:257260.

19. Raison JS, Bourbotte G, Baum TP, Pagès M. [primary brain stem hemorrhage: Retrospective study of 25 cases]. Rev Neurol (Paris) 2008;164:225-232.

20. Kumral E, Bayam FE, Özerol R, Orman M. Predictors of outcome in patients with medullary hemorrhage. J Stroke Cerebrovasc Dis 2020;29:105337.

21. Chotai S, Qi S, Xu S. Prediction of outcomes for brainstem cavernous malformation. Clin Neurol Neurosurg 2013;115:21172123.

22. Chung CS, Park CH. Primary pontine hemorrhage: a new ct classification. Neurology 1992;42:830-834.

23. Dziewas R, Kremer M, Lüdemann P, Nabavi DG, Dräger B, Ringelstein B. The prognostic impact of clinical and ct parameters in patients with pontine hemorrhage. Cerebrovasc Dis 2003;16:224-229. 
24. Ikeda K, Kawakami K, Onimaru H, Okada Y, Yokota S, Koshiya $\mathrm{N}$, et al. The respiratory control mechanisms in the brainstem and spinal cord: Integrative views of the neuroanatomy and neurophysiology.J Physiol Sci 2017;67:45-62.

25. Bonis JM, Neumueller SE, Krause KL, Kiner T, Smith A, Marshall $\mathrm{BD}$, et al. The pontine respiratory group, particularly the kölliker-fuse nucleus, mediates phases of the hypoxic ventilatory response in unanesthetized goats. J Appl Physiol (1985) 2010;108:1321-1335.

26. Bolton CF, Chen R, Wijdicks EFM, Zifko UA. Neurology of breathing. Butterworth-Heinemann; 2004. 